\title{
Contenção de onça pintada (Panthera onca) para Avaliação de possível miíase na região peitoral
}

\author{
Containment of jaguar ( anthera onca) for Assessment of possible myiasis in the pectoral region \\ Contención de jaguar (Panthera onca) para evaluación de posible miasis en la región pectoral
}

Recebido: 19/08/2021 | Revisado: 29/08/2021 | Aceito: 26/12/2021 | Publicado: 05/01/2022

\author{
Thiago Vaz Lopes \\ ORCID: https://orcid.org/0000-0002-3192-1908 \\ Universidade Federal do Acre, Brasil \\ E-mail: thiagovlopes@ hotmail.com \\ João Gustavo da Silva Garcia de Souza \\ ORCID: https://orcid.org/0000-0002-5899-2490 \\ Centro Universitário Aparício Carvalho, Brasil \\ E-mail: joaogarciadesouza27@gmail.com \\ Joana Morais \\ ORCID: https://orcid.org/0000-0001-9448-375X \\ Centro Universitário Aparício Carvalho, Brasil \\ E-mail: joanamoraismed.vet@gmail.com \\ Igor Mansur Muniz \\ ORCID: https://orcid.org/0000-0003-0863-6647 \\ Centro Universitário Aparício Carvalho, Brasil \\ E-mail: igor.mansur@unir.br \\ Sandro de Vargas Schons \\ ORCID: https://orcid.org/0000-0001-9811-5356 \\ Universidade Federal de Rondônia, Brasil \\ E-mail: sandroschons@unir.br \\ Fernando Andrade Souza \\ ORCID: https://orcid.org/0000-0002-9474-9404 \\ Universidade Federal do Paraná, Curitiba, Brasil \\ E-mail: femedvet@yahoo.com.br
}

\begin{abstract}
Resumo
A onça pintada encontra-se em status de vulnerabilidade e está ameaçada de extinção. Muitos espécimes se encontram em estado de conservação em locais como zoológicos e viveiros de felinos silvestres, com o intuito de proteger felinos que se encontram em estado de impossibilidade de voltar a natureza. O objetivo desse trabalho foi de relatar uma contenção de Panthera onca para avaliação de possível miíase em dois ferimentos. Uma onça pintada de aproximadamente 20 anos de idade, fêmea, coloração amarelo claro, seu condicionamento físico score corporal foram também avaliados. Foi observado por seus tratadores uma lesão na região peitoral e axilar, com a possível presença de mí́ase em ambos. Foi também pedido uma avaliação bioquímica dos perfis renais e hepáticos. Como resultados, observou-se a presença de lesões sugestivas de recidiva da neoplasia, presença de miíase nos locais e feito uma reavaliação após 10 dias do tratamento. Os bioquímicos demonstraram discretas alterações. Foi possível concluir que a contenção química destes animais é muito benéfica, visto que protege a integridade física tanto do animal, quanto da equipe de veterinários e auxiliares.
\end{abstract}

Pavalvras chave: Panthera onca; Contenção; Silvestres; Anestesia.

\begin{abstract}
The jaguar is in a vulnerable status and is threatened with extinction. Many specimens are in a state of conservation in places such as zoos and wild cat nurseries, in order to protect cats that are unable to return to nature. The objective of this work was to report a Panthera onca retainer for the evaluation of possible myiasis in two wounds. An approximately 20 -year-old female jaguar, light yellow in color, and its physical fitness score were also evaluated. His caretakers observed a lesion in the pectoral and axillary regions, with the possible presence of myiasis in both. A biochemical assessment of kidney and liver profiles was also requested. As a result, the presence of lesions suggestive of cancer recurrence, the presence of myiasis in the sites were observed, and a reassessment was carried out after 10 days of treatment. Biochemists showed slight changes. It was possible to conclude that the chemical containment of these animals is very beneficial, as it protects the physical integrity of both the animal and the team of veterinarians and assistants.
\end{abstract}

Keywords: Panthera onca; Containment; Wild; Anesthesia. 


\section{Resumen}

El jaguar se encuentra en un estado vulnerable y está amenazado de extinción. Muchos ejemplares se encuentran en estado de conservación en lugares como zoológicos y viveros de gatos salvajes, con el fin de proteger a los gatos que no pueden volver a la naturaleza. El objetivo de este estudio fue reportar un retenedor de Panthera onca para la evaluación de una posible miasis en dos heridas. También se evaluó una hembra de jaguar de aproximadamente 20 años, de color amarillo claro, y su puntaje de aptitud física. Sus cuidadores observaron una lesión en las regiones pectoral y axilar, con posible presencia de miasis en ambas. También se solicitó una evaluación bioquímica de los perfiles renal y hepático. Como resultado, se observó la presencia de lesiones sugestivas de recidiva de neoplasias, la presencia de miasis en los sitios y se realizó una reevaluación a los 10 días de tratamiento. Los bioquímicos mostraron ligeros cambios. Se pudo concluir que la contención química de estos animales es muy beneficiosa, ya que protege la integridad física tanto del animal como del equipo de veterinarios y asistentes.

Palabras clave: Panthera onca; Contención; Salvaje; Anestesia.

\section{Introdução}

A onça pintada pertence ao Filo: Chordata; Classe: Mamalia; Ordem: Carnívora; Família: Felidae; Gênero e espécie: Panthera onca (Linneaus,1758), é o único representante deste gênero no continente americano. Esta espécie se encontra em um status de conservação entre vulnerável a ameaçada em extinção conforme sua distribuição geográfica (ICMBIO, 2020).

A conservação de algumas espécies silvestres tem sido alvo de discussões e controvérsia em relação aos zoológicos e instituições protetivas (Bressan; Kierulff; Sugieda, 2009), pois dentro da temática zoológico/instituições conservacionistas, existe vários fatores que possam ser discutidos em relação ao bem estar animal, maneira de preservação e ideias de educação ambiental, visando contribuir para a manutenção dos animais, ameaçados ou vulneráveis, ao avanço das atividades humana, pois a ausência destes animais desequilibra todo um ecossistema como exemplo a onça pintada e outros grandes felinos, tem-se gerado um grande esforço a nível internacional para realizar atividades de educação ambiental e fortalecer ações relacionadas a conservação da biodiversidade (Miller et al. 2004; Waza, 2005).

Em grandes felídeos, sejam livres ou restritos em ambientes de conservação, para realização de procedimentos médicos que envolva a manipulação desses animais por humanos, podendo gerar desconforto para o bem estar animal, e risco para equipe envolvida, sendo assim se faz necessário o uso de fármacos pra contenção, tornado possível a aproximação com segurança, sendo a equipe sempre constituída por médicos veterinários e biólogos (Chaves \& Abmussi, 2015; Souza et al. 2021), permitindo assim avaliação física, (Fiorello et al. 2007), colheita de materiais biológicos, (Wenger et al. 2010), procedimentos de diagnósticos e terapêuticos (Schrader et al. 2012).

Esses animais possuem hábitos solitários, mas costumam interagir com indivíduos de gênero oposto, por questões territoriais ou reprodutivos, quando em reprodução, a gestação dura de 90 a 100 dias, com o nascimento de um a quatro filhotes. Os felídeos brasileiros são encontrados em uma grande diversidade de habitats, estão distribuídos em todos os biomas com exceção dos campos do Sul (Cavalcanti et al. 2012; Oliveira, Ramalho, De Paula, 2012) além das florestas são também encontrados em ecossistemas como cerrado, caatinga e pantanal (Morato et. al. 2013). Em vida livre estima-se uma duração de vida de até 15 anos, quando criados em cativeiro a expectativa de vida pode aumentar em até 10 anos. As presas desses amimais são bem variáveis, desde grandes animais como as antas, porcos-do-mato, jacarés, bicho preguiça, capivaras entre outros a pequenos animais como jabutis, peixes, macacos, aves e até sapos (ICMBIO, 2020).

Atualmente a onça-pintada é descrita mundialmente como espécie quase ameaçada de extinção pela União Internacional para a Conservação da Natureza. A espécie no Brasil e catalogada como vulnerável pelo livro vermelho da fauna brasileira ameaçada de extinção (ICMBIO, 2018). Em alguns biomas brasileiros as populações da espécie se encontram reduzida gravemente (Ferraz et al. 2012).

A Amazônia brasileira alberga menos de 10.000 indivíduos em seus 3.459 .000 km2, na Amazônia e no Pantanal encontramse os maiores números de indivíduos de onça-pintada de todo o mundo (Cavalcanti et al. 2012; Oliveira, Ramalho, De Paula, 2012). As percepções e ações humanas estão diretamente ligadas e determinam a permanência, conservação e continuidade desta 
espécie no Brasil e em todos as áreas de atuação. (Stokes, 2007; Marchini \& Macdonald, 2012). Sendo assim este relato tem como objetivo descrever uma contenção química por neurolepetoanalgesia em onça pintada em Zoológico Militar.

\section{Relato de Caso}

A onça pintada denominada pertencente à Família Felidae, Gênero Panthera e Espécie Panthera Onca (Onça pintada) com idade de aproximadamente 20 anos, fêmea, coloração amarelo claro, cujo na avaliação física foi observado seu condicionamento físico, score corporal. Como queixa principal, foi observado por seus tratadores lesão na região peitoral e axilar com possível presença de miíase, o felino tem por histórico a manifestação de hemangiossarcoma, sendo esse diagnóstico já pré-estabelecido através da avaliação histopatológica. Para a avaliação do estado geral do animal foram colheitados materiais biológicos para a realização de exames complementares como mensuração da glicemia, hemograma, bioquímicos para perfil renal e hepático (Ureia, Creatinina, ALT, FA e Glicose) como também avaliação físico-química da urina.

\section{Resultados e Discussão}

As colheitas de material biológico para avaliação do quadro geral do animal, foi realizado afim de aproveitar ao máximo de o processo de sedação e para que se pudesse fechar um diagnostico de ligação de aleterações sistêmicas com ligação a manifestação da lesão cutânea. Durante a avaliação pode-se observar a presença de lesões sugestivas de recidiva da neoplasia, para que fosse possível então a realização dessa colheita de material biológico, avaliação física e limpeza do ferimento com presença de mí́ase. Foi então efetuado tratamento de retirada de miíase, administração de ivermectina $0,4 \mathrm{mg} / \mathrm{kg}$, limpeza da ferida, aplicação de pomada cicatrizante e administração de Carprofeno durante 10 dias, e sugerido uma reavaliação após tratamento o término do tratamento.

Após dez dias de início do tratamento clínico e da primeira contenção química, foi então realizada a segunda contenção para uma reavaliação. Para a essa contenção foi estabelecido aplicação de anestésicos em associação, sendo realizada com o uso de zarabatana e dardo com colarinho, o animal já se encontrava em espaço um espaço reduzido, na jaula de contenção, presente no recinto, descartando o risco de lesão e estresse, por já estar devidamente adaptado a ela. O protocolo utilizado teve como objetivo tranquilização rápida, segura, estabilidade cardiovascular e recuperação sem eventos inesperados. Após contenção e neuroleptoanalgesia animal foi levado à sala cirúrgica, colocado em mesa cirúrgica para anestesia volátil, mantendo-o em plano anestésico.

Os fármacos utilizados na MPA foram associação de Meperidina (opióide) $11 \mathrm{mg} / \mathrm{kg}$ com Dexmedetomidina (agonista a2 adrenérgico) $0,03 \mathrm{mg} / \mathrm{kg}$, realizado então a indução anestésica e manutenção do plano, animal pesando $38,5 \mathrm{~kg}$, apresentando então mucosas oral, ocular e vulvar normocoradas, temperatura retal $36,8 \mathrm{c}^{\circ}$ (referência $37^{\circ}-39,5^{\circ}$ ). Em relação ao grau de hidratação, estava normohidratada, com olhos brilhantes, na avaliação de pele e anexos foram observadas lesões na região peitoral e escaras no crânio próximo aos olhos, como também a presença de pequena úlcera na boca. Na ausculta cardíaca 72 BPM (referência 80 - 16 bpm); na verificação nos movimentos respiratórios 24 MPM (referência 8 - 24 MPM) (Rocha, 2009; Vieira, 2021).

Para a manutenção anestésica a onça pintada foi colocada em uma mesa com calha cirúrgica, para indução anestésica e intubação endotraqueal, seguindo protocolo de verificação de manutenção dos sinais vitais. Para indução de plano anestésico utilizou-se Cetamina (anestésico dissociativo) 2-5mg/kg IV associado com Midazolam (benzodiazepínico) 0,3 mg/kg, e como manutenção foi utilizado o anestésico volátil isofluorano 1,5\% como fluxo de $\mathrm{O} 2$ com 500ml/minuto. 
Seguidamente destes procedimentos, foi avaliado clinicamente e minuciosamente as lesões que o animal apresentava na pele, lesões estas, superficiais com características de boa cicatrização sem tecido necrosado ou presença, as lesões se encontravam em aspecto limpo sem secreção purulenta e com tecido de granulação. Realizado então limpeza delas com água oxigenada e clorexidine degermante, administrado por via tópica pomada cicatrizante Alantolß, sendo descartada a recidiva de hemangiossarcoma por avaliação clínica das lesões.

Todo o processo teve a duração de 5 horas, entre a contenção química inicial e o retorno da paciente novamente a gaiola de contenção, com o restabelecimento das condições motoras e psíquicas, a paciente foi acompanhada a distância, por seu tratador durante o retorno anestésico, não apresentando nenhuma alteração ou excitação, somente após restabelecidos seus reflexos motores e psíquicos ela foi então liberada a área livre do recinto, esse que é divido com outra onça pintada do sexo oposto.

Para a contenção foi realizado o dardejamento de acordo com Gunkel \& Lafortune (2007) ao explicar a importância de uma jaula de contenção para evitar trauma após administração dos fármacos, sendo realizado a neuroleptonalagesia conforme descrito na literatura, por associação de um opióide e um agonista a2-adrenérgico, sendo os de escolha para tal a Meperidina e Dexmedetomidina, respectivamente, concordando com Massone (2003) e Fantoni (2009), alcançando o relaxamento muscular, uma tranquilização e analgesia satisfatória.

A escolha desses fármacos forneceu segurança para o paciente, pois o mesmo é um animal sênior que precisa de cuidados redobrados, em relação à estabilidade respiratória e cardíaca, sendo assim possível uma avaliação física tranquila, completa e segura, a escolha da meperidina, foi bem aceita pois Da Costa (2014) relata a sua rapidez e eficiência em procedimentos não demorados e que necessitam de um retorno tranquilo do paciente. Esta associação contou então com o uso de um representante agonistas a2-adrenérgico, completando o conceito de neuroleptoanalgesia dito por Massone (2003) \& Fantoni (2009) o fármaco de escolha foi a dexmedetomidina por suas propriedades e especificidades aos receptores alfa2, diminuindo as secreções gastrointestinais e ações diuréticas, conforme Plumb \& Pharm (2010).

Os resultados dos exames laboratoriais demonstraram discretas alterações, assim como o encontrado por Thrall (2006) na avaliação sérica da ALT (Alanina Aminotransferase), foi evidenciado um aumento, caracterizando lesão hepática focal ou multifocal. assim como houve aumento na série branca do hemograma com neutrófilia, eosinófilia e monócitose supondo um quadro de parasitismo, inflamação e até mesmo devido ao seu histórico, um processo de neoplasia, ou até mesmo ligado as lesões cutâneas indo de encontro com que afirmaram Gonzales \& Da Silva (2008).

$\mathrm{Na}$ avaliação da urina apresentou padrões dentro da normalidade para a espécie, com exceção dos valores de densidade no grau de 1018\% (referência 1015\% a 1030\%) sendo esse maior que o esperado para a espécie, sendo que Kerr (2003) afirma que a urinálise não é o padrão ouro para detecção de doença renal crônica ou aguda, porém é de grande importância como exame de triagem, porem sempre quando avaliado deve ser ponderado e considerado o tipo de alimentação, peso, ingestão de água, para uma correta interpretação desse exame.

\section{Conclusão}

A contenção química dos grandes felinos selvagens, é uma realidade, sejam eles de vida livre ou em cativeiro, torna possível e segura a realização de procedimentos como o relatado, garantindo a integridade do animal e da equipe envolvida.

\section{Referências}

Bressan, P. M., Kierulff, M. C. M. \& Sugieda, A. M. (2009). Fauna ameaçada de extinção no estado de são paulo: vertebrados. Parque Zoológico de São Paulo: Secretaria do Meio Ambiente.

Cavalcanti, S. M. C. et al. (2012). The status of the jaguar in the Pantanal.Cat News, Special p. 29-34.

Chaves, A. S. Y. R. \& Abimussi, C. J. X. (2015). Contenção de grandes felinos - Revisão de literatura containment wild felids - Review. Alm. Med. Vet. Zoo. 2015 . 
Research, Society and Development, v. 11, n. 1, e23211119613, 2022

(CC BY 4.0) | ISSN 2525-3409 | DOI: http://dx.doi.org/10.33448/rsd-v11i1.19613

Costa, P. F. et al. (2014). Parâmetros ventilométricos e hemogasométricos em cadelas submetidas à ovariohisterectomia, pré-medicadas com tramadol ou morfina e anestesiadas com isofluorano. Revista Brasileira de Ciência Veterinária.

Fantoni, D. T. \& Cortopassi, S. R. (2009). Anestesia em Cães e Gatos. São Paulo: 2ed., Roca.

Ferraz, K. M. P. M. B. et al. (2012). How species distribution models can improve cat conservation-jaguars in Brazil. Cat News, Special, 7: 38-42.

Fiorello, C. V. et al. (2007). Diagnosis and treatment of presumptive postobstructive pulmonary edema in a Florida panther (Puma concolor coryi). Journal of Zoo and Wildlife Medicine.

Gunkel, C. \& Lafortune, M. (2007). Felids. In West, G.; Heard, D. \& Caulkett, N. (Eds.), Zoo Animal and Wildlife Immobilization and Anesthesia. Ames, Iowa, USA: Blackwell Publishing, 2007.

ICMBIO- Instituto Chico Mendes de Conservação da Biodiversidade. (2013). Plano de ação nacional para conservação da onça-pintada. Brasília.

ICMBIO (Ed.). (2018). Livro Vermelho da Fauna Brasileira Ameaçada de Extinção: s. Brasilia: Instituto Chico Mendes de Conservação da Biodiversidade.

Miller, B. et al. (2004). Evaluating the Conservation Mission of Zoos, Aquariums, Botanical Gardens, and Natural History Museums. Conservation Biology, 18(1): $1-8$.

Marchini, S. \& Macdonald, D. W. (2012). Predicting ranchers' intention to kill jaguars: case studies in Amazonia and Pantanal. Biological Conservation, 147(1): $213-21$.

Massone, F. (2003). Aplicações parenterais. In: medicações pré-anestésicas; Atlas de anestesiologia veterinária. São Paulo: Roca.

Morato, R. G. et al. (2013). Avaliação do risco de extinção da Onça-pintada Panthera onca (Linnaeus, 1758) no Brasil. Instituto Chico Mendes de Conservação da Biodiversidade, Brasil.

Oliveira, T. G., Ramalho, E. E. \& De Paula, R. C. (2012). Red list assessment of the jaguar in Brazilian Amazonia. Cat News, Bern, Special, 7: 8-13.

Rocha, V. M. (2009). Parâmetros eletrocardiográficos de felideos selvagens da amazônia (phantera onxa, leopardus pardalis, leopardus wiedii, herpailurus yaguarondi)) criados em cativeiro no estado do pará, tranquilizados com ketamina e cloridrato de xilazina. 93f. Dissertação de conclusão de curso Pósgraduação em Medicina Veterinária, na área de Ciência Animal. Belém, Pará.

Schrader, G. M. et al. (2012). Conservative management of pyothorax in an Amur tiger (Panthera tigris altaica). Journal of Zoo and Wildlife Medicine.

Souza, M. V. et al. (2021). Contenção farmacológica de onças-pardas puma concolor (Carnivora: felidae) com tiletamina, zolazepam, detomidina, atropina, com ou sem azaperone, em doses calculadas por extrapolação alométrica interespecífica. Pensar Acadêmico, 19(1): 1-20.

Stokes, D. L. (2007). Things we like: human preferences among similar organisms and implications for conservation. Human Ecology, 35(3): 361-369.

Vieira, A. C. (2021). Avaliação do padrão eletrocardiográfico em onças-pintadas (Panthera Onca) em vida livre no pantanal sul mato-grossense. Dissertação de conclusão de curso em Medicina Veterinária. https://repositorio.animaeducacao.com.br/handle/ANIMA/13798

Waza. (2005). Construindo um Futuro para a Vida Selvagem: Estratégia Mundial dos Zoos e Aquários para a Conservação. Peter J. S. Olney (Ed.). Tradução: Jardim Zoológico e de Aclimação em Portugal S.A, Lisboa, Portugal.

Wenger, S. et al. (2010). Evaluation of Butorphanol, Medetomidine and Midazolam as a Reversible Narcotic Combination in Free-Ranging African Lions (Panthera Leo). 